\title{
A survey of video capsule endoscope system based on wireless energy transfer technology
}

\author{
Ting Fang* and Xiongwei $\mathrm{Wu}$ \\ Nanchang Institute of Technology, Nanchang, China
}

Keywords: Capsule endoscope, Wireless, Energy transfer.

\begin{abstract}
The wireless capsule endoscope system allows clinicians to directly observe the image of the inner wall of the human gastrointestinal tract and obtain the most intuitive information at the lesion. Compared with traditional endoscopic detection technology, its painless, non-invasive, safe and convenient, full gastrointestinal detection features make this technology a research focus in the field of medical devices at home and abroad. As a new non-invasive detection technology for gastrointestinal diseases, the working time, image frame rate and image quality of the existing wireless capsule endoscope system cannot fully meet the needs in clinical applications. The cause of these problems lies in the capsule. The internal space of the speculum is limited, and only the button battery can be used as the energy source. Therefore, it is necessary to design a video capsule endoscope based on wireless energy transmission technology. This article is a review of Zhu Zhanquan, Xue Kaifeng, Lu Ruiqi and other scholars.
\end{abstract}

\section{Introduction}

Human beings have been unremittingly exploring their own mysteries. Exploring the human digestive tract is not only to uncover the mystery of the human body, but also to meet the urgent needs of people's healthy life. Digestive tract function is the source of energy and nutrients required by the human body to achieve the absorption and utilization of nutrients required by the human body. With the development of society, the pace of life is accelerating, and the human diet has undergone corresponding changes, making the incidence of digestive tract diseases appear to be on the rise. At present, the incidence of gastrointestinal diseases accounts for $20 \%$ of the world's total population, and the probability of cancer is higher. The number of new cases of intestinal cancer in China has reached 160,000 per year.

Many dangerous diseases are most easily cured in the early stages of the disease. Gastrointestinal bleeding is a very common digestive tract disease and a complication of many digestive diseases. In order to detect gastrointestinal bleeding, many indirect detection methods such as angiography, CT, X-ray imaging and ultrasound scanning imaging have been gradually developed. However, it has been found in the clinic that the

*Corresponding author: nclgdz@,foxmail.com 
diagnostic rate of the above detection method is only about $5 \%$, and the detection techniques such as X-ray imaging, ultrasound scanning imaging, and angiography have thresholds for the detection of blood loss, and only the bleeding per minute at the patient's lesion. The amount is more than $3 \mathrm{ml}$, and the effect of scintillation is limited. For the diagnosis and treatment of digestive diseases, traditional endoscopes such as gastroscopy and colonoscopy are relatively common and relatively effective methods. However, at present, the traditional endoscope is directly inserted from the patient's mouth or anus, and the optical fiber, image signal line, power line, etc. are integrated in a soft pipe, which not only causes a huge problem for the patient in the diagnosis process. Pain, but also has a chance to cause related complications. In addition, the traditional endoscope has a "blind zone" for detection. The human digestive tract has a long and narrow physiological structure. It is difficult to achieve long-distance insertion by using a catheter, so that the small intestine located in the middle of the digestive tract cannot be detected.

\section{The status quo of wireless energy supply capsule endoscopy}

With the advancement of technology, and benefiting from the rapid development of semiconductor technology, communication technology, integrated circuits and MEMS technology, it can enter the human gastrointestinal tract, and realize the painless, noninvasive or minimally invasive diagnosis and treatment technology has become a hot spot in international biomedical research. . The proposed image capsule endoscope has made it possible to see its replacement for a conventional insertion endoscope. The image capsule endoscope is shaped like a common pill. It integrates a lighting system, a micro imaging system, a wireless communication system and a micro control unit. It can work continuously for about 8 hours, and is swallowed by the patient's gastrointestinal tract after swallowing. The image of the lining of the gastrointestinal tract is taken at a frame rate of $2 \mathrm{f} / \mathrm{s}$ until the battery is exhausted or discharged from the body by the anus. However, in the later clinical stage, the image capsule endoscope exposed the shortcomings of low acquisition frame rate and limited working time. The cause of these problems lies in its energy bottleneck. The image capsule endoscope is only powered by two button batteries very limited. In recent years, wireless energy transmission technology is also one of the research hotspots. It uses the electromagnetic field as the medium to realize the wireless transmission of electric energy. It has been widely used in electric vehicle charging, small mobile electronic device charging and power supply of implantable medical equipment. Research and application, this wireless power supply method provides a new idea for breaking the energy bottleneck of image capsule endoscope.

At present, the working principle of capsule endoscopes is basically the same on the market, but its frame rate is greatly affected by wireless communication technology and energy limitation. According to the data, the size of an image collected by the existing capsule endoscope is about $70 \mathrm{k}$. If the acquisition frame rate is $\mathrm{mf} / \mathrm{s}$, the wireless transmission module will transmit 70k data per second, which requires higher bandwidth for wireless communication. At the same time, the larger the amount of data transmitted, the higher the energy consumption of the communication module, which greatly shortens the effective working time of the capsule endoscope. Therefore, the working principle of the existing capsule endoscope hinders the development of the capsule endoscope in a multifunctional, video, and clearer direction.

Due to the size and working process of the micro-diagnostic device, the application of wireless energy-supplied technology in this field has certain specialties and technical difficulties. Judging from the research results obtained by the wireless energy supply technology of micro-diagnostic devices at home and abroad, although different degrees of progress have been made in various aspects, these studies are still in a preliminary and 
partial exploration stage, which is far from perfect. Systematic theoretical results still have a certain distance. There is no convincing theoretical derivation and experimental verification for the energy transmission efficiency problem under weak coupling conditions; the structural size of the transmitting coil and the receiving coil, and the selection of the winding turns and the shape size of the magnetic core have no theoretical basis; human tissue The safety problem in the strong magnetic field environment has attracted people's attention, but its relationship with the structure of the transmitting coil and the transmitting power is not compared and analyzed by an overall system. Therefore, wireless energy supply for solving the energy bottleneck problem of capsule endoscope still requires systematic and in-depth research.

\section{The key problem of video capsule endoscope system research based on wireless energy transmission technology}

Based on the analysis of the current research status of capsule endoscope, the purpose of this study is a video capsule endoscope system based on wireless energy transmission technology, and Verification by experimental testing. The technical research of video capsule endoscope based on wireless energy transmission can be divided into two parts: video capsule endoscope technology and wireless energy transmission technology; in these two technologies, electromagnetic field technology is also involved; the former uses electromagnetic waves to realize video. The wireless transmission of signals uses electromagnetic fields to achieve a wireless supply of system power. Therefore, in view of the video capsule endoscope application and the special physiological environment of human gastrointestinal tract detection, it is first necessary to study the interaction between the electromagnetic field and the human body. The specific content of the study is:

1. Analyze and study the interaction between electromagnetic fields and human bodies. Study the coupling mechanism between human tissue and electromagnetic field, establish a high-precision human body electromagnetic calculation model, and obtain the relationship between electromagnetic dose and frequency and emission current of different tissues of human body.

2. Analyze the problems encountered in the design of video capsule endoscopes and possible solutions, and propose and design an in vitro video image receiving system that matches the video capsule endoscope technology, to achieve the reception, storage, and repetition of video images. Play, while its real-time display function can monitor the working state of the video capsule endoscope.

3. Propose the video capsule endoscope wireless energy transmission system scheme and related control strategies to establish a generalized space model. On the basis of the model, the uncertainty and parameter perturbation of the energy transmission system are analyzed and researched.

4. Based on the experimental research, through the combination of data analysis and theory, the parameter optimization and function verification of the wireless energy supply video capsule endoscope system are carried out.

\section{The technical route of video capsule endoscope system based on wireless energy transmission technology}

Through the literature and field investigation to identify the production and development process of capsule endoscope, analysis of its clinical manifestations due to energy bottlenecks Summarize the research results and the experience of wireless energy transmission technology in the application of micro-diagnostic devices in recent years. 
Study the interaction between electromagnetic fields and human bodies. Firstly, the coupling mechanism between human tissue and electromagnetic field should be studied. The electromagnetic parameter data of different tissues of different human tissues can be obtained through calculation. Then, based on the color photograph of cross-section of VHP male human body, a high-precision human electromagnetic calculation model is established. . The human body electromagnetic calculation model can first analyze the propagation of electromagnetic waves transmitted by wireless signals in a loss medium, and then calculate the power loss caused by human body propagation; then according to the international safety standards for human exposure to electromagnetic fields and The guideline can clarify the induced current density and specific absorption rate SAR as the main monitoring parameters of low-frequency biological tissue safety, and calculate the electromagnetic parameters in human tissue under different frequency and different emission current conditions, from the perspective of safety. The foundation for the design and optimization of wireless energy transmission systems [1].

A specific design scheme of a high-rate video capsule endoscope system is proposed. First of all, we must study the physiological characteristics of the human gastrointestinal tract. It is necessary to meet the requirements of the capsule design model for the working environment of the video capsule endoscope. Then, for the high-speed image acquisition requirements, the video image subsystem, control subsystem and wireless communication subsystem inside the capsule are studied and designed. Compare the image sensor manufacturing process, select the image sensor based on function, size and energy consumption, and then configure the corresponding lens and lighting module according to the sensor characteristics of the model; design the communication configuration protocol of the image sensor, select the most satisfying function The simplified micro-control chip realizes the low-power image sensor working mode configuration; for the video transmission mode, it is necessary to select the radio frequency modulation mode with high energy utilization rate, strong anti-interference ability and large communication data volume to realize wireless video transmission, thereby Design the corresponding RF transmitting circuit and the RF transmitting antenna suitable for the structure of the capsule endoscope of this video, and then carry out simulation analysis and verification separately. Finally, the structure and function of the external video image receiving system can be designed[1].

Research on wireless energy transmission technology. Through the analysis of the wireless energy transmission mode and the technical principle of electromagnetic induction, this study intends to use the inductive coupling energy transmission technology to realize the wireless power supply of the system. Aiming at the requirements of inductive coupled energy transmission technology, combined with the specific working conditions of video capsule endoscope, the video capsule endoscope wireless energy transmission system scheme and related control strategies are proposed, and then the loosely coupled transformer model and the improved generalized space model are established, which will be System optimization analysis and stability design provide theoretical basis. Based on the system model, the uncertainty of system parameters is analyzed and researched, and the corresponding quantitative standards and methods are proposed.

On the basis of experimental research, parameter optimization and function verification of the wireless energy supply video capsule endoscope system are also required. In the experiment of video acquisition part, it is necessary to test the image sensor lighting module and the wireless communication module separately, compare the theoretical analysis results with the actual measurement results, establish the working voltage, and ensure the illumination requirements of the frequency capsule endoscope and the wireless communication chain. Road power margin. The correctness of the video acquisition part system design can be verified by the intestine test in the intestine; the parameters of the 
transmitter end can be obtained through the experiment of the energy acquisition part; the experimental data can be obtained through experimental comparison. The receiving coil model of the video capsule endoscope; using the robust stability analysis method, a stable wireless energy transmission system can be established. Finally, animal live experiments were carried out to verify the performance of the system $[1,3]$.

\section{Summary}

This study will aim at the imperfection of biological electromagnetic dose experimental measurement methods, establish a high-precision human body electromagnetic simulation model, propose a human body electromagnetic parameter calculation method, and provide a quantitative research platform for human body electromagnetic dose calculation. During the research, modeling and analyzing the propagation loss of electromagnetic waves in human media provides a basis for the calculation of wireless communication link margin; it will study the safety of human tissue in electromagnetic field environment, which can be the biosafety of wireless energy transmission system. Aiming at the stability problem and weak coupling problem of wireless energy transmission, an inductive coupled energy transmission system model is proposed, and a wireless energy transmission system model applied to video capsule endoscope is established. A parameter optimization model and optimization are proposed for energy transmission stability method. Through experimental research, it is possible to establish a comprehensive system optimized design scheme for the energy transmitting end and the receiving end. Provide ideas for the study of wireless energy transmission technology stability and transmission efficiency[2].

This paper is the research result of the three-dimensional magnetic coupling inductive radio energy transmission technology research (GJJ161166) of the science and technology project of Jiangxi Provincial Department of Education.

\section{References}

1. Jiang Ning, Fan Yihong, Meng Lina et al. Clinical application analysis of domestic OMOM capsule endoscopy [J]. Chinese Journal of Digestive Endoscopy, 2007.

2. Xie Xiang, Li Guolin, Zhang Chun et al. Design of a two-way digital micro wireless endoscope system [J]. Research and Development of Solid State Electronics, 2007.

3. Xie Xiang, Li Guolin, Chi Baoyong et al. Design of digital-analog hybrid circuit in two-way and digital micro-radio endoscope capsules [J]. Journal of Tsinghua University, 2005. 